Ocular Oncology

and Pathology
Ocul Oncol Pathol 2018;4:186-190

DOI: $10.1159 / 000481509$
Received: June 7, 2017

Accepted after revision: September 13, 2017

Published online: November 10, 2017

\title{
Malignant Orbital Meningioma Originating from the Frontal Lobe
}

\author{
Giancarlo A. Garcia $^{a}$ Andrew E. Choy ${ }^{b, c}$ Anton N. Hasso ${ }^{d}$ Don S. Minckler ${ }^{a}$ \\ ${ }^{a}$ Gavin Herbert Eye Institute, University of California, Irvine, Irvine, $C A,{ }^{b}$ Family Eye Medical Group, and \\ 'Long Beach Memorial Hospital, Long Beach, CA, and d Department of Radiological Sciences, University of \\ California Irvine Medical Center, Orange, CA, USA
}

\section{Established Facts}

- Orbital meningiomas are uncommon and usually benign tumors, typically arising from the sphenoid bone or optic nerve sheath.

\section{Novel Insights}

- An anaplastic orbital meningioma can originate from the frontal lobe of the brain, and can be associated with orbital and distant extracranial metastases.

- Orbital invasion may be more likely after surgical resection of aggressive frontal lobe meningiomas.

\section{Keywords}

Meningioma · Orbit · Craniotomy · Tumor recurrence ·

Metastasis · Exenteration · Anaplastic features

\begin{abstract}
Purpose of the Study: Orbital meningiomas are typically benign tumors, most commonly originating from the dura of the sphenoid wing or the optic nerve sheath. Procedures: We describe an unusual case of a malignant meningioma originating from the frontal lobe that ultimately produced orbital and distant metastases. Results and Conclusions: Orbital invasion by the meningioma was preceded by multiple incomplete resections, which may have facilitated access to the orbit. The present case serves to remind clinicians that surgical resection of aggressive, recurrent
\end{abstract}

\section{KARGER}

(c) 2017 S. Karger AG, Basel

E-Mail karger@karger.com

www.karger.com/oop frontal lobe meningiomas may facilitate subsequent penetration of surrounding structures, particularly by tumors that demonstrate bone-destructive properties.

(c) 2017 S. Karger AG, Basel

\section{Introduction}

Meningiomas are the most common intracranial neoplasms, typically occurring in the fourth through sixth decade of life [1]. They are usually benign, indolent tumors, twice as prevalent in women as men, with fewer

This case was presented in part at the Verhoeff-Zimmerman meeting at Wills Eye Hospital, Philadelphia, PA, on April 22, 2017.
Giancarlo A. Garcia, MD, and Don S. Minckler, MD

Gavin Herbert Eye Institute, University of California, Irvine 850 Health Sciences Road

Irvine, CA 92697 (USA)

E-Mail garcia.giancar@gmail.com 
Fig. 1. Coronal (a) and sagittal (b) enhanced CT scans showing an entirely intracranial dural-based mass in the right frontal pole with no extension into the orbital roof or frontal bone. There is no involvement of the sinuses or the falx. There is considerable surrounding edema in the frontal lobe.
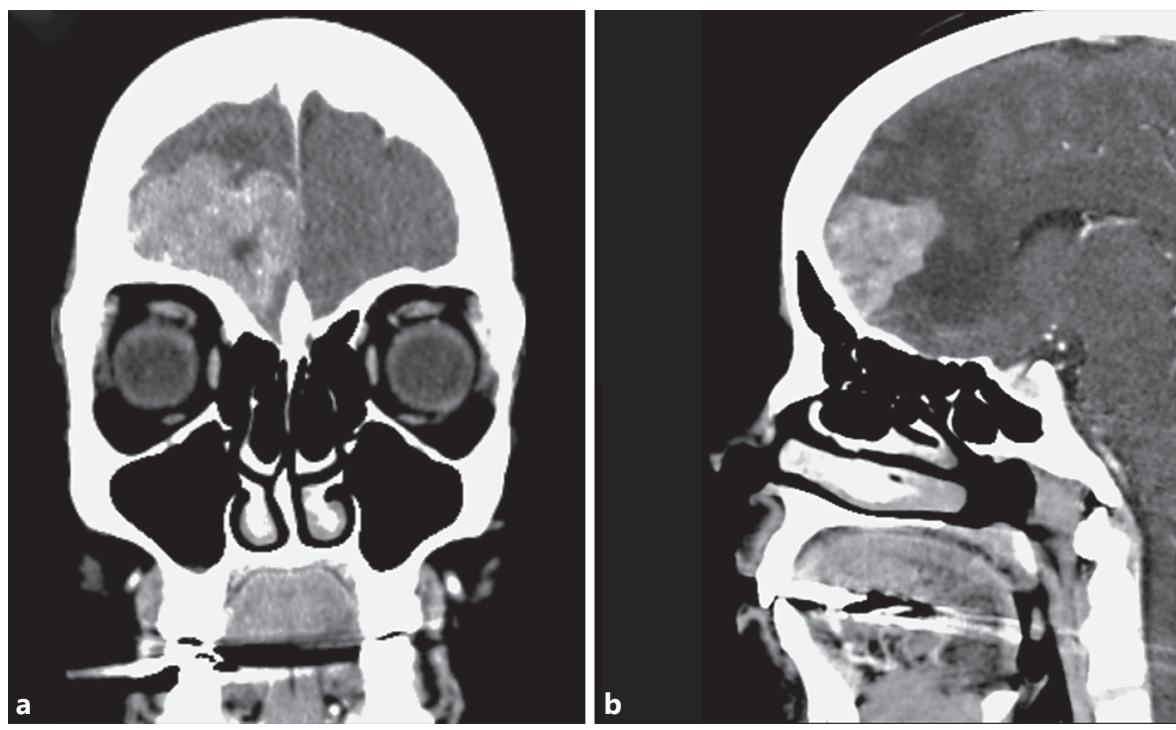

than $10 \%$ demonstrating anaplastic features or distant metastases [1,2].

Orbital meningiomas are estimated to account for $3-9 \%$ of all intraorbital neoplasms [3, 4]. Sphenoidal-origin meningiomas often narrow the optic canal and compress the optic nerve at that location. Primary orbital optic nerve meningiomas typically compress the nerve as they expand in the subdural space, and present with progressive, unilateral vision loss, axial proptosis, and optic atrophy. Involvement of the anterior optic nerve may be associated with disc shunt vessels visible ophthalmoscopically. Clinical features of sphenoidal or other-origin intracranial meningiomas include, headaches, nausea and vomiting, and papilledema.

Primary orbital meningiomas arise from the optic nerve sheath meningothelial cells or rarely from ectopic arachnoid tissue, whereas secondary orbital meningiomas most commonly originate from the sphenoid wing [5]. Herein, we describe an unusual case of an orbital meningioma originating from the frontal lobe convexity, additionally remarkable for its aggressive behavior, anaplastic histology, multiple recurrences, local tissue destruction, and extracranial metastases.

\section{Case Report}

A 54-year-old African-American woman was referred for an oculoplastic and orbital surgery consultation for orbital involvement by an anaplastic-subtype convexity meningioma of the right frontal lobe diagnosed 14 years prior. Initial recognition of the tumor had been preceded by recurrent headaches and sei- zures. Interval therapy had included 4 craniotomies, all demonstrating incomplete excision. Radiation therapy after the initial excision and following a subsequent excision were judged nonbeneficial. Medical therapy had included levetiracetam and zolpidem.

Resection \#2, 12 years after resection \#1, was performed with right frontal bone craniotomy and cranioplasty and demonstrated a $5.5 \times 1 \mathrm{~cm}$ right frontal convexity dural-based mass extending along the right skull base, without orbital or sinus involvement (Fig. 1). Two additional resections were performed ( $\# 3$ and $\# 4$ ) at intervals thereafter of 5 and 14 months, respectively. Residual right frontal lobe tumor was noted on MRI after each surgery. Histopathology after each resection demonstrated a World Health Organization (WHO) grade III anaplastic meningioma with Ki-67 positive tumor cells, consistent with recurrence of the patient's previously incompletely excised tumor. An MRI performed 5 months after resection \#4, requested by neurosurgery to track CNS tumor size for possible re-resection, demonstrated the first radiologic evidence of orbital involvement, extending through the roof of the right orbit into the superior space. Although no intraocular involvement was evident (Fig. 2a), the tumor displaced the globe inferiorly and temporally. The patient then came under ophthalmic plastics care for further evaluation and treatment of the orbital involvement.

At her plastics consultation, the patient complained of rightsided orbital pain and profound diminution in visual acuity in the right eye. She had no other past ocular history or prior ophthalmic surgeries, and had no prior ophthalmic examinations for comparison. On examination, a firm right upper lid mass was present measuring $40 \mathrm{~mm}$ in the largest dimension. There was prominent proptosis of the right globe and marked ptosis of the right eyelid (Fig. 2b). Visual acuity was light perception OD, which improved to 20/25 with manual elevation of the right eyelid, and 20/20 OS. The right eye demonstrated a relative afferent pupillary defect, and the left pupillary reflex was normal. Intraocular pressures were 24 $\mathrm{OD}$ and $18 \mathrm{~mm} \mathrm{Hg} \mathrm{OS}$. Confrontational visual field testing showed defects in all 4 quadrants OD and no defects OS. Slit lamp exami- 

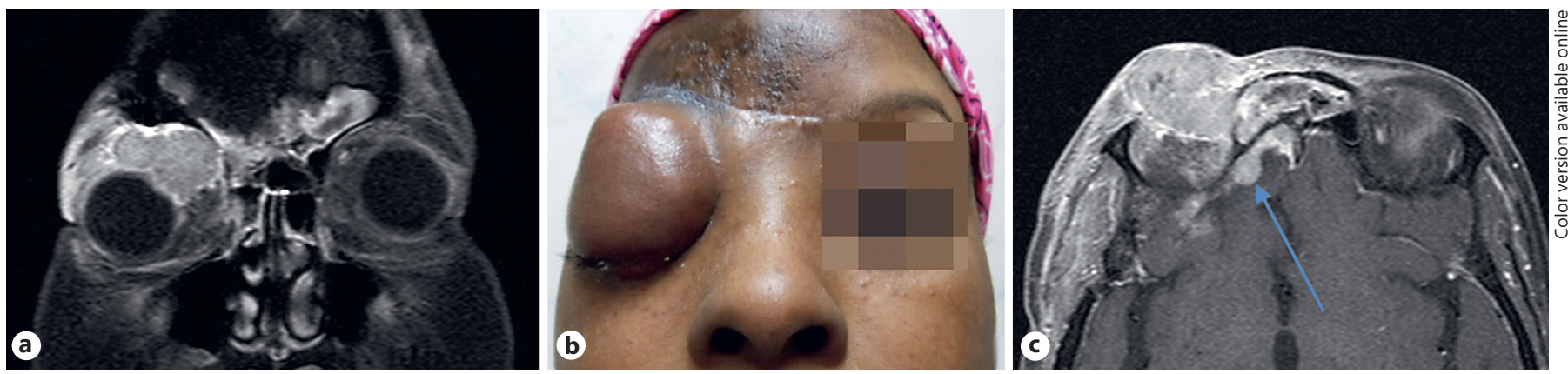

Fig. 2. a Coronal MRI scan (T1-weighted following gadolinium contrast infusion) performed after 4 tumor resection surgeries. There is a lobulated enhancing extraconal tumor mass located in the right superior orbit compressing the globe inferiorly and temporally. No intraocular invasion is evident. b Facial photograph of the patient demonstrating a firm right upper lid mass measuring $40 \mathrm{~mm}$ in the largest dimension. There is prominent right-sided proptosis and near-complete ptosis. c Axial MRI scan (T1-weight-

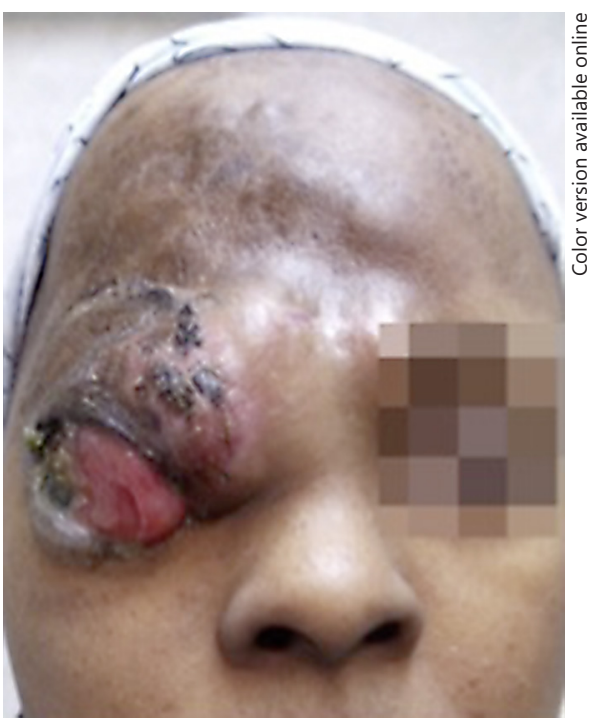

Fig. 3. Facial photograph of the patient 2 months after a subtotal tumor resection was performed, demonstrating worsening proptosis and orbital deformity, purulent discharge, persistent granulation tissue, and cutaneous erythema and scab formation.

nation of the anterior and posterior segments was normal bilaterally. An additional MRI performed 6 months after the previous MRI demonstrated more extensive orbital invasion, prominent involvement of the right frontal sinus, and loss of the cortical bony margin (Fig. 2c). The tumor had infiltrated the superior and medial rectus muscles, as well as the superior oblique muscle and tendon and broached the orbital septum with subsequent progressive lid involvement.

A right orbitotomy was performed to biopsy and characterize the orbital mass. Tumor tissue was sampled from the superior na- ed fat-saturated images following gadolinium contrast infusion) performed 6 months after the scan depicted in a. The enhancing combined intra- (arrow) and extracranial tumor mass is well seen. The extracranial component extends into the superior medial portion of the orbit and displaces the globe temporally. There is tumor in the intervening frontal sinus as well with loss of the cortical bony margin.

sal orbit, along with partial resection of the superior rectus muscle. Intraoperatively, it was apparent that the tumor had extensively infiltrated the soft tissue structures of the right orbit and, accordingly, total removal of the orbital mass could not be assured and therefore was not attempted. Histopathology confirmed that the orbital mass was an anaplastic meningioma consistent with prior pathology.

Two months after the orbital biopsy, the patient experienced intractable right eye and orbit pain poorly controlled by analgesics, worsening proptosis and orbital deformity, purulent discharge, persistent granulation tissue, and cutaneous erythema and scab formation (Fig. 3). A right orbital exenteration was subsequently performed as a palliative measure to alleviate these symptoms. Intraoperatively, exploration revealed that the tumor had eroded through the right frontal sinus and infiltrated the nasal two-thirds of the orbit displacing the globe inferiorly and temporally.

Pathologic examination of the exenteration specimen confirmed previous pathology and a diffusely infiltrating tumor of the orbit and eyelids. Tumor cells were pleomorphic with large vesicular nuclei, abundant eosinophilic cytoplasm, and frequent mitoses (Fig. 4). Immunohistochemical staining of the orbital tumor was positive for CD68, epithelial membrane antigen, moderately high proliferation index (Ki-67), progesterone receptor, and somatostatin receptor type 2 . The tumor was present in the conjunctival stroma and eyelid dermis. Sections of the globe demonstrated reduction in retinal ganglion cells and early optic atrophy but no scleral or intraocular tumor.

Two months after the exenteration, the patient began experiencing dyspnea, hemoptysis, and chest pain. CT of the chest, abdomen, and pelvis demonstrated multiple well-defined intrapulmonary nodules bilaterally, confirmed by CT core biopsy of the left lung as anaplastic meningioma with histology again consistent with metastatic anaplastic meningioma (Fig. 4). External iliac lymph nodes were also prominent on imaging but not biopsied. The patient's clinical condition subsequently deteriorated and she died from respiratory impairment 5 months later while in hospice care. 


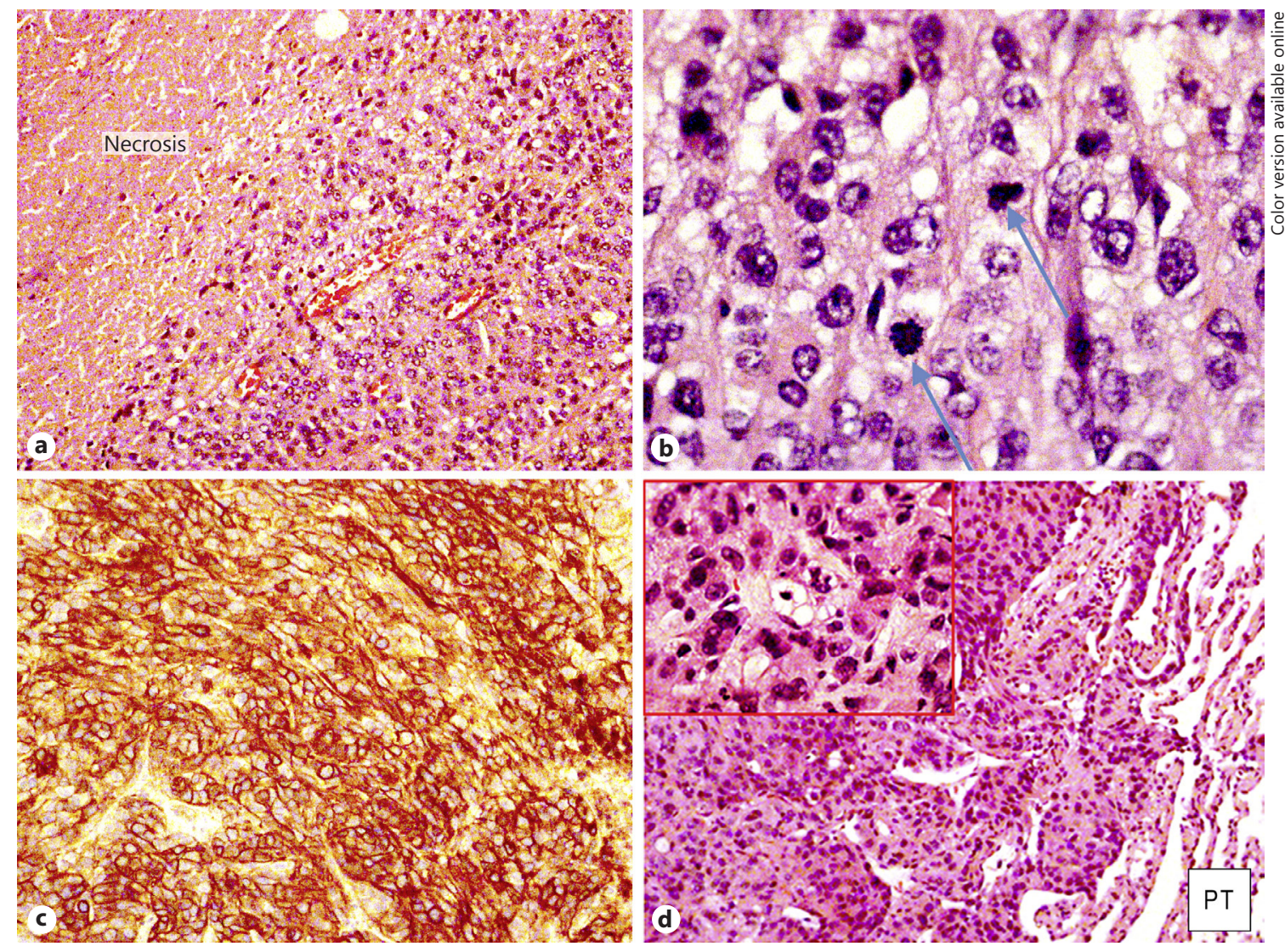

Fig. 4. a Photomicrograph of orbital extension of frontal lobe malignant meningioma demonstrating margin between tumor tissue that is viable (lower right side) and necrotic (upper left side, labeled). HE. Original magnification $\times 125$. b Higher-magnification photomicrograph of orbital meningioma demonstrating pleomorphic tumor cells with large vesicular nuclei and prominent nucleoli, including 2 abnormal mitoses (arrows). HE. Original magnification $\times 500$. c Photomicrograph of IHC somatostatin- 2 which is highly positive in the orbital lesion and consistent with meningioma. Somatostatin receptor type 2 . Original magnification $\times 125$. d Photomicrograph of lung metastasis biopsy (CT core sample) from left lung including the margin of pulmonary tissue (PT) on the right. HE. Original magnification $\times 125$. The inset at higher magnification $(\mathrm{HE}$; original magnification $\times 500$ ) demonstrates an area of the same biopsy with histologic characteristics similar to those in the orbital lesion.

\section{Discussion}

The present case demonstrates a secondary orbital malignant meningioma originating from a highly unusual primary site, the frontal lobe convexity.

The mechanism of orbital invasion in this case is uncertain, although the patient's history of multiple craniotomies preceding the first radiographic evidence of orbital involvement could have played a significant role. The patient underwent a total of 4 frontal bone craniotomies before orbital involvement became apparent. Highly malignant intracranial tumors such as glioblastoma multiforme have been observed to infiltrate the orbit through a prior craniotomy site [6-8], although, to our knowledge, invasion of the orbit by a meningioma through a craniotomy site has not been proven. The possibility of hematogenous spread from the frontal convexity to the orbit is less likely in this case, given the presence of only a single mass in the orbit rather than multiple distinct foci, and strong imaging evidence of direct extension.

Alternatively, the tumor may have penetrated the orbit by gross destruction of orbital bone. Malignant meningioma subtypes may exhibit bony destruction or hyperostosis [9]. Sphenoid wing meningiomas, in particular, demonstrate a high incidence of bone involvement [10], and cases of intraosseous meningiomas can display local bone destruction. Extension into the orbit through bone has also been previously reported in cases of glioblastoma multiforme, and, in rare cases, by pituitary tumors and 
craniopharyngiomas [11]. Interestingly, various reports describe the phenomenon of convexity meningiomas eroding through bone in the absence of prior craniotomies, though not into the orbit specifically $[12,13]$.

An additional peculiarity of this case is the highly malignant histology and aggressive behavior of the meningioma, with multiple recurrences likely due to incomplete excision, and eventual metastasis to the lungs and iliac nodes. Meningiomas arising over the convexities of the brain are generally benign, and typically have high potential for complete surgical excision, if a substantial margin of excision can be achieved. However, those with anaplastic pathology, as in the present case, can demonstrate 5 -year recurrence rates as high as 50\% [14].

Meningiomas are rarely metastatic, with distant extracranial metastases estimated to occur in approximately $0.001 \%$ of cases, most commonly to the lung [15]. As expected, the tumor described in this case exhibited characteristics associated with a higher risk for metastasis, including histologic malignancy (WHO grade III), and local recurrences. Adlakha et al. [15] in 1999 suggested that previous craniotomy may be a predisposing factor for metastasis, although the mechanism underlying this association has not been clearly elucidated.
In conclusion, this case illustrates a secondary orbital meningioma arising from an unusual site of origin, the frontal lobe. Though the mechanism of orbital invasion in this case is uncertain, clinicians should be aware that a history of multiple craniotomies to resect aggressive frontal lobe meningiomas may confer a higher risk of both orbital involvement and subsequent metastases.

\section{Statement of Ethics}

The IRB at UCI does not require a one-patient clinicopathologic case report to include informed consent.

\section{Disclosure Statement}

The authors have no competing or conflicting interests. The authors have no disclosures.

\section{Funding Sources}

The Department of Ophthalmology at University of California, Irvine, is a recipient of an institutional Research to Prevent Blindness Unrestricted Grant.

\section{References}

1 Ali AN, Travers K, Athavale N, Al-Modoaris F: Malignant meningioma - a rare cause of temporal head lump. QJM 2014;107:833-835.

2 Kalamarides M, Goutagny S: Meningiomas. Rev Prat 2006;56:1792-1798.

3 Reese AB: Tumors of the Eye, ed 3. Hagerstown, Harper \& Row, 1976, pp 148-153.

4 Henderson JW, Farrow GM: Orbital Tumors, ed 2. New York, BC Decker, 1980, pp 472496.

5 Boulos PT, Dumont AS, Mandell JW, Jane JA Sr: Meningiomas of the orbit: contemporary considerations. Neurosurg Focus 2001;10:E5.

6 Aoyama I, Makita Y, Nabeshima S, et al: Extradural nasal and orbital extension of glioblastoma multiforme without previous surgical intervention. Surg Neurol 1980;14:343347.
7 Hoyt WF, Piovenetti E, Malmud N, et al: Cranio-orbital involvement in glioblastoma multiforme. Neurochirugia 1972;15:1-8.

8 Lawton AW, Karesh JW: Intracranial glioblastoma invading the orbit. Arch Ophthalmol 1986;104:806.

9 Hsu C, Pai C, Kao H, Hsueh C, Hsu W, Lo C: Do aggressive imaging features correlate with advanced histopathological grade in meningiomas? J Clin Neurosci 2010;17:584-587.

10 Pompili A, Derome PJ, Visot A, et al: Hyperostosing menigiomas of the sphenoid ridge clinical features, surgical therapy, and longterm observations: review of 49 cases. Surg Neurol 1982;17:411-416.
11 Shinder R, Esmaeli B: Secondary orbital tumors extending from ocular or periorbital structures; in Esmaeli B (ed): Ophthalmic Oncology. New York, Springer, 2011, pp 79-87.

12 Kaminski JM, Movsas B, King E, et al: Metastatic meningioma to the lung with multiple pleural metastases. Am J Clin Oncol 2001;24: 579-582.

13 Velnar T, Pregelj R, Limbaeck-Stokin C: Brain meningioma invading and destructing the skull bone: replacement of the missing bone in vivo. Radiol Oncol 2011;45:304-309.

14 Morokoff AP, Zauberman J, Black PM: Surgery for convexity meningiomas. Neurosurgery 2008;63:427-433; discussion 433-434.

15 Adlakha A, Rao K, Adlakha H, et al: Meningioma metastatic to the lung. Mayo Clin Proc 1999;74:1129-1133. 